\title{
KLOE results on neutral kaon decays
}

\author{
KLOE Collaboration* \\ presented by Matthew Moulson ${ }^{\dagger}$ \\ INFN Frascati, Italy \\ E-mail: Matthew.Moulson@lnf.infn.it
}

The KLOE experiment at the Frascati $\phi$ factory DA $\Phi N E$ collected $450 \mathrm{pb}^{-1}$ of data during 20012002 running, and is expected to have collected an additional $2 \mathrm{fb}^{-1}$ by the end of this year. This report summarizes recently completed results on neutral kaon decays based on the 2001-2002 data, including branching ratio measurements for $K_{S} \rightarrow 2 \pi$ and $K_{S} \rightarrow 3 \pi$ decays, semileptonic $K_{S}$ decays, and the dominant $K_{L}$ decays; measurements of the $K_{L}$ lifetime; and measurements of the form-factor slopes for $K_{e 3}$ decays of the $K_{L}$. Prospects for improvements and new results based on the 2004-2005 data are also described.

International Europhysics Conference on High Energy Physics

July 21st - 27th 2005

Lisboa, Portugal

${ }^{*}$ F. Ambrosino, A. Antonelli, M. Antonelli, C. Bacci, P. Beltrame, G. Bencivenni, S. Bertolucci, C. Bini, C. Bloise, V. Bocci, F. Bossi, D. Bowring, P. Branchini, R. Caloi, P. Campana, G. Capon, T. Capussela, F. Ceradini, S. Chi, G. Chiefari, P. Ciambrone, S. Conetti, E. De Lucia, P. De Simone, G. De Zorzi, S. Dell'Agnello, A. Denig, A. Di Domenico, C. Di Donato, S. Di Falco, B. Di Micco, A. Doria, M. Dreucci, G. Felici, A. Ferrari, M. L. Ferrer, G. Finocchiaro, C. Forti, P. Franzini, C. Gatti, P. Gauzzi, S. Giovannella, E. Gorini, E. Graziani, M. Incagli, W. Kluge, V. Kulikov, F. Lacava, G. Lanfranchi, J. Lee-Franzini, D. Leone, M. Martini, P. Massarotti, W. Mei, S. Meola, S. Miscetti, M. Moulson, S. Müller, F. Murtas, M. Napolitano, F. Nguyen, M. Palutan, E. Pasqualucci, A. Passeri, V. Patera, F. Perfetto, L. Pontecorvo, M. Primavera, P. Santangelo, E. Santovetti, G. Saracino, B. Sciascia, A. Sciubba, F. Scuri, I. Sfiligoi, A. Sibidanov, T. Spadaro, M. Testa, L. Tortora, P. Valente, B. Valeriani, G. Venanzoni, S. Veneziano, A. Ventura, R. Versaci, G. Xu.

†Speaker. 


\section{KLOE and DA $\Phi N E$}

KLOE is a large, general-purpose experiment at the Frascati $\phi$ factory, DAФNE, an $e^{+} e^{-}$ collider running at $\sqrt{s}=m_{\phi} \approx 1019.5 \mathrm{MeV}$. During the years 2001-2002, KLOE collected a data set of about $450 \mathrm{pb}^{-1}$. After a DAФNE shutdown for upgrades in 2003, KLOE data taking restarted in spring 2004. KLOE will end running at $\sqrt{s}=m_{\phi}$ at the end of 2005, with an expected 2004-2005 data set of about $2 \mathrm{fb}^{-1}$.

The KLOE detector consists of a cylindrical drift chamber (DC), surrounded by an electromagnetic calorimeter $(\mathrm{EmC})$. A superconducting coil provides a $0.52 \mathrm{~T}$ axial magnetic field. The drift chamber [1] is $4 \mathrm{~m}$ in diameter and $3.3 \mathrm{~m}$ long. Tracks from the origin with $\theta>40^{\circ}$ are reconstructed with $\sigma_{p} / p \leq 0.4 \%$. The energy resolution of the lead/scintillating-fiber calorimeter [2] is $\sigma_{E} / E=5.7 \% / \sqrt{ } E(\mathrm{GeV})$. The stochastic contribution to the time resolution is $\sigma_{t}=$ $54 \mathrm{ps} / \sqrt{ } E(\mathrm{GeV})$. Photon vertices from $\pi^{0}$ decays inside the DC (e.g., from $K_{L} \rightarrow \pi^{+} \pi^{-} \pi^{0}$ ) are reconstructed by time of flight (TOF) with a resolution of about $2 \mathrm{~cm}$.

The KLOE Monte Carlo (MC) [3] includes a detailed description of the detector, and includes two features of special interest for analyses of neutral kaon decays. First, machine background is extracted from the data set and overlaid with generated events. This is particularly important in searches for rare $K_{S}$ decays. Second, the kaon decay generators implement radiative contributions as described in Ref. 4. This is important for all precision measurements of $K_{S}$ and $K_{L}$ decays.

\section{KLOE and the $K_{S} K_{L}$ system: Tagging and interference measurements}

At a $\phi$ factory such as DA $\Phi$ NE, about $10^{6} K_{S} K_{L}$ pairs are produced per $\mathrm{pb}^{-1}$ of integrated luminosity. These pairs are in a pure, antisymmetric quantum state $\left(J^{P C}=1^{--}\right)$. Reconstruction of a $K_{S}$ decay in an event therefore signals the presence of a $K_{L}$ and vice versa. Most KLOE measurements of the $K_{S} K_{L}$ system are "tagged" measurements based on this principle.

Since the $K_{S}$ and $K_{L}$ are initially in a pure quantum state, it is also possible to observe the effects of quantum-mechanical interference. When the two kaons decay to final states $f_{1}$ and $f_{2}$ at times $t_{1}$ and $t_{2}$, the decay distribution in $\Delta t=t_{2}-t_{1}$ contains an interference term proportional to $\cos \left(\Delta m \Delta t-\phi_{12}\right)$, where $\Delta m=m_{L}-m_{S}$, and $\phi_{12}$ is the phase difference between the amplitude ratios $\eta_{1,2}$ for $K_{L}$ and $K_{S}$ decays to each final state. This allows measurement of the $C P$ parameters $\eta$ and other quantities [5]. When $f_{1}=f_{2}, \phi_{12}=0$, i.e., because of the antisymmetry of the initial state and the symmetry of the final state, there should be no events with $\Delta t=0$. This has been demonstrated using $380 \mathrm{pb}^{-1}$ of KLOE data from 2001-2002. The distribution in $\Delta t$ for $K_{S} K_{L} \rightarrow \pi^{+} \pi^{-} \pi^{+} \pi^{-}$ events was formed and fit with a function incorporating the experimental resolution and the effect of $K_{L} \rightarrow K_{S}$ regeneration on the beam pipe, in addition to the expected modulation of frequency $\Delta m$ (Fig. 1). The interference term in the fit function was multiplied by $\left(1-\zeta_{S L}\right)$, where normal quantum mechanics predicts $\zeta_{S L}=0$. With $\Delta m$ fixed to the PDG value [6], KLOE obtains the preliminary result $\zeta_{S L}=0.043_{-0.035}^{+0.038} \pm 0.008$, which is consistent with zero and a factor of four more stringent as a test of quantum mechanics than the result based on an analysis of CPLEAR data on the $K^{0} \bar{K}^{0}$ system [7]. 


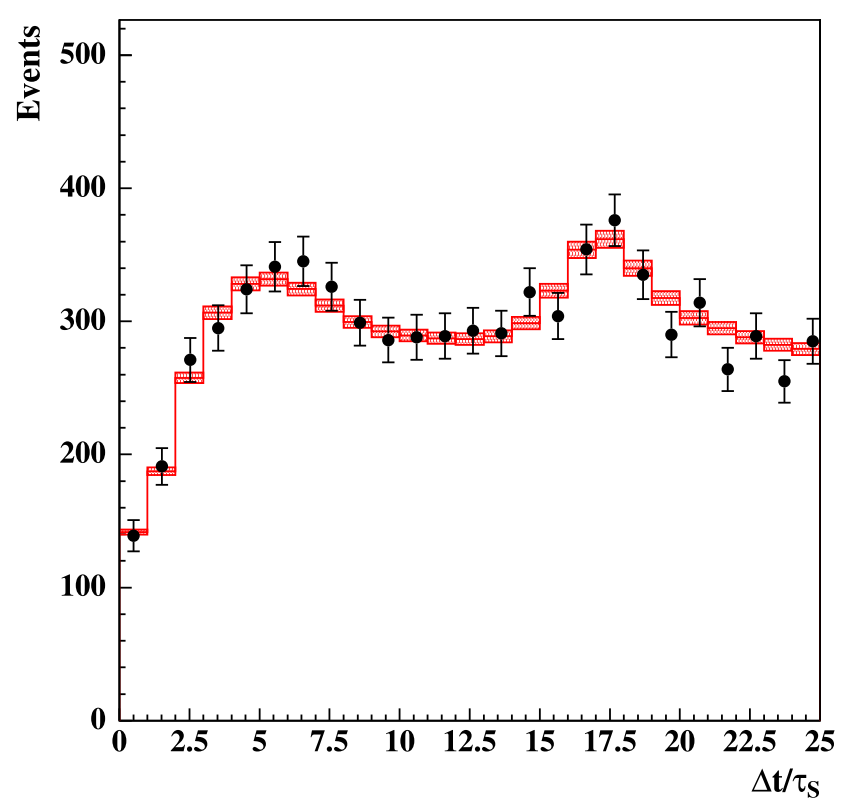

Figure 1: Distribution in $\Delta t$ for $\phi \rightarrow K_{S} K_{L} \rightarrow \pi^{+} \pi^{-} \pi^{+} \pi^{-}$events. Points are data; red line indicates fit. The deficit at $\Delta t=0$ and the modulation of frequency $\Delta m$ resulting in the peak at $\Delta t / \tau_{S} \approx 5$ demonstrate the effects of quantum-mechanical interference. The excess at $\Delta t / \tau_{S} \approx 18$ arises from $K_{L} \rightarrow K_{S}$ regeneration on the beam pipe.

\section{3. $K_{S}$ decays}

$K_{S}$ decays are tagged by the interaction of a $K_{L}$ in the EmC, referred to as a " $K_{L}$ crash." The $K_{L}$ crash is recognized as an isolated, high-energy (typically, $E>100 \mathrm{MeV}$ ) cluster that arrives roughly $30 \mathrm{~ns}$ after the clusters from the $K_{S}$ decay. The tagging efficiency is about $30 \%$ and is dominated by the probability for the $K_{L}$ to reach the calorimeter. The position of the $K_{L}$ crash, together with the kinematics of the $\phi \rightarrow K_{S} K_{L}$ decay, determines the trajectory of the $K_{S}$ a priori with a momentum resolution of about $1 \mathrm{MeV}$ and an angular resolution better than $1^{\circ}$. The simulation of the EmC response to the $K_{L}$ crash in the KLOE MC has been carefully adjusted with reference to data [8].

A measurement of the ratio $R_{\pi \pi} \equiv \operatorname{BR}\left(K_{S} \rightarrow \pi^{+} \pi^{-}(\gamma)\right) / \operatorname{BR}\left(K_{S} \rightarrow \pi^{0} \pi^{0}\right)$ fixes the value of $\operatorname{BR}\left(K_{S} \rightarrow \pi^{+} \pi^{-}(\gamma)\right)$, which normalizes branching ratio measurements of other $K_{S}$ decays. KLOE also uses the analysis of $R_{\pi \pi}$ as a laboratory for the reduction of experimental systematics. The ratio itself is interesting in that it can be used to determine $\delta_{0}-\delta_{2}$, the difference in strong finalstate $\pi \pi$ phase shifts (see, e.g., Ref. 9). KLOE has non-zero acceptance over the entire range of photon energies for $K_{S} \rightarrow \pi^{+} \pi^{-} \gamma$ decays, and the KLOE measurement of $R_{\pi \pi}$ is fully inclusive of such decays. The previous KLOE measurement of $R_{\pi \pi}$ [10], based on $17 \mathrm{pb}^{-1}$ of year 2000 data, was characterized by a $0.7 \%$ error dominated by systematics. The analysis has been repeated using the 2001-2002 data set $\left(410 \mathrm{pb}^{-1}\right)$, with various improvements. The preliminary value obtained for $R_{\pi \pi}$ is $2.256 \pm 0.003 \pm 0.010$. A further reduction of the $0.4 \%$ systematic error is expected as this result is finalized.

The decay $K_{S} \rightarrow 3 \pi^{0}$ is purely $C P$ violating. If $C P T$ is conserved, the BR for this decay can be predicted from $\Gamma_{S}=\Gamma_{L}\left|\varepsilon+\varepsilon_{000}^{\prime}\right|^{2} \approx 1.9 \times 10^{-9}$. In KLOE, the signature is an event with a $K_{L}$ 

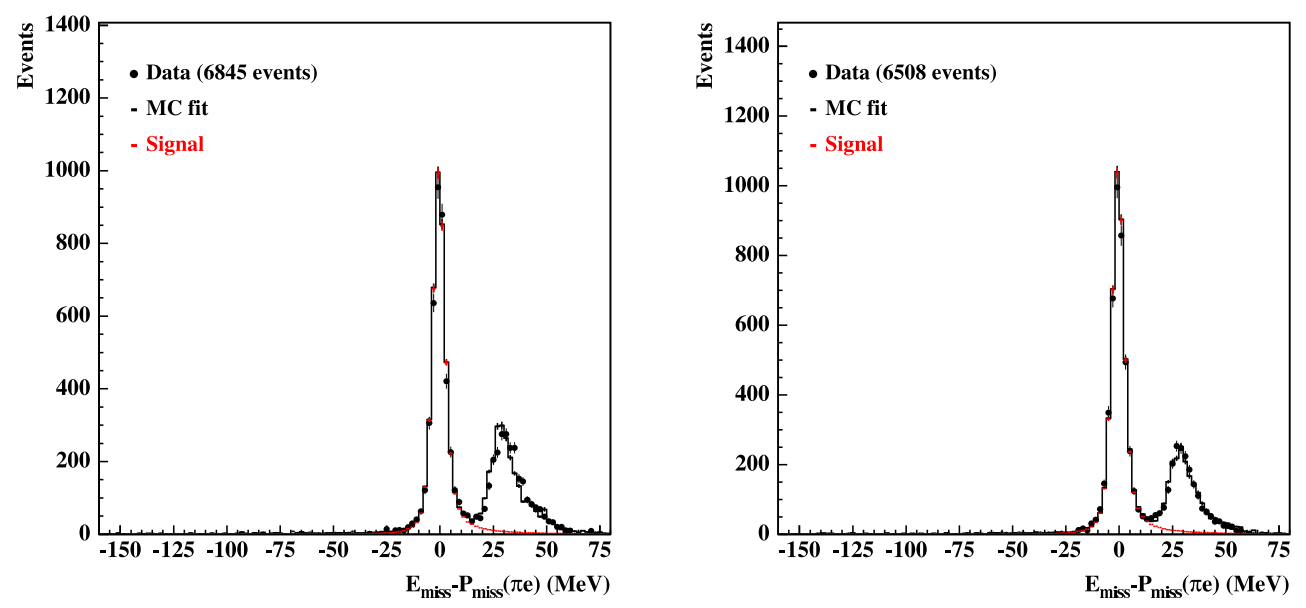

Figure 2: Distributions in $E_{\text {miss }}-p_{\text {miss }}$ (evaluated in the signal mass hypothesis) for candidate $K_{S} \rightarrow \pi e v$ events in the 2002 data set. Left panel: $\pi^{-} e^{+} \nu$ events. Right panel: $\pi^{+} e^{-} \bar{v}$ events.

crash, six photon clusters, and no tracks from the interaction point. Background is mainly from $K_{S} \rightarrow \pi^{0} \pi^{0}$ events with two spurious clusters from splittings or accidental activity. Based on an analysis of $450 \mathrm{pb}^{-1}$ of 2001-2002 data, KLOE has obtained the $90 \%$ CL limit BR $\leq 1.2 \times 10^{-7}$ [11]. With the additional $2 \mathrm{fb}^{-1}$ of data from 2004-2005 and improvements to the analysis under development, this limit can potentially be reduced by an additional order of magnitude. KLOE can also use the $K_{L^{-}}$crash tagged sample to search for the decay $K_{S} \rightarrow \pi^{+} \pi^{-} \pi^{0}$, which proceeds mainly via a $C P$-conserving, $\Delta I=3 / 2$ transition. The current PDG value of the $\mathrm{BR}$ for this decay, $3.2_{-1.0}^{+1.2} \times 10^{-7}$, is computed from $K_{S} K_{L}$ interference measurements. KLOE has used $740 \mathrm{pb}^{-1}$ of data from 2001, 2002, and 2004 to estimate the precision on the BR that can be obtained in a direct search. Six candidate events are identified with an estimated background of 3.5 events. With $2 \mathrm{fb}^{-1}$, KLOE should be able to measure the BR for this decay with a $60 \%$ error, i.e., with precision similar to that of the interference-based measurements currently entering the PDG average.

The semileptonic decays of the neutral kaons can be used to test $C P T$ through the charge asymmetries $A_{S, L}$, defined as the difference between the widths for $K_{S}$ or $K_{L}$ decays to final states of each lepton charge, divided by their sum. $A_{S}$ and $A_{L}$ quantify $C P$ violation in $K^{0} \bar{K}^{0}$ mixing, and should both be equal to $2 \operatorname{Re} \varepsilon$. If $A_{S}$ and $A_{L}$ are found to be different, $C P T$ is violated, either in $K^{0} \bar{K}^{0}$ mixing or in direct transitions with $\Delta S \neq \Delta Q$. The equivalence of the partial widths for semileptonic $K_{S}$ and $K_{L}$ decays is also a test of the $\Delta S=\Delta Q$ rule. Finally, the CKM matrix element $V_{u s}$ can be determined from the semileptonic widths. The current PDG value for the BR is dominated by the KLOE measurement made with $17 \mathrm{pb}^{-1}$ of year 2000 data [12], which has an error of about $5 \%$ with no distinction between charge states. The KLOE analysis of $K_{S} \rightarrow \pi e v$ proceeds from the $K_{L}$-crash tagged sample and exploits the excellent timing performance of the EmC to identify signal events by TOF and to assign the charge of the lepton in the final state. Figure 2 shows the distributions in $E_{\text {miss }}-p_{\text {miss }}$ for $K_{S} \rightarrow \pi e v$ event candidates for each state of lepton charge. This quantity is similar to the missing mass and is zero for signal events. The signal peak is prominent and cleanly separated from the background. Using $410 \mathrm{pb}^{-1}$ of data from 2001 and 2002, KLOE has obtained the preliminary results $\operatorname{BR}\left(\pi^{-} e^{+} v\right)=(3.53 \pm 0.06 \pm 0.03) \times 10^{-4}$, 
$\mathrm{BR}\left(\pi^{+} e^{-} \bar{v}\right)=(3.52 \pm 0.05 \pm 0.03) \times 10^{-4}$, and $\operatorname{BR}(\pi e v)=(7.05 \pm 0.08 \pm 0.05) \times 10^{-4}$. The value of the combined BR for both charge states is in good agreement both with the previous $\mathrm{KLOE}$ value, and with the $\mathrm{KLOE}$ (see below) and $\mathrm{KTeV}$ [13] values for $\mathrm{BR}\left(K_{L} \rightarrow \pi e v\right)$, assuming $\Delta S=\Delta Q$ and using the KLOE average value for $\tau_{L}$ (the $K_{L}$ lifetime) discussed below. $A_{S}$ is found to be $(1.5 \pm 9.6 \pm 2.9) \times 10^{-3}$. With the full $2.5 \mathrm{fb}^{-1}$ from all running, KLOE expects to measure $A_{S}$ with an uncertainty of $\sim 3 \times 10^{-3}$, about equal to the expected value of this parameter. Measurement of $\operatorname{BR}\left(K_{S} \rightarrow \pi \mu v\right)$ is more difficult, as the similarity of the $\pi$ and $\mu$ masses complicates TOF PID, and background from $K_{S} \rightarrow \pi^{+} \pi^{-}$decays with $\pi \rightarrow \mu v$ is harder to eliminate. Nevertheless, a preliminary analysis has been performed on the 2001-2002 data, and yields $\operatorname{BR}\left(K_{S} \rightarrow \pi \mu v\right)$ with a $3 \%$ statistical error.

\section{4. $K_{L}$ decays}

The BR's for $K_{L}$ decays to $\pi e v$ and $\pi \mu \nu$ give access to $V_{u s}$. Heretofore, these BR's were known only through various measurements of the ratios of the $K_{L}$ BR's for dominant decays (i.e., decays to $3 \pi^{0}$ and $\pi^{+} \pi^{-} \pi^{0}$, in addition to the above). Using the $K_{S} \rightarrow \pi^{+} \pi^{-}$decay as a tag, KLOE has measured the absolute BR's for all four dominant decays with uncertainties of 0.5$1 \%$ [14]. The analysis is based on $328 \mathrm{pb}^{-1}$ of 2001-2002 data. Decays to $\pi e v, \pi \mu \nu$, and $\pi^{+} \pi^{-} \pi^{0}$ are reconstructed in the DC with PID from the decay kinematics. Decays to $3 \pi^{0}$ are reconstructed in the EmC as TOF-localized vertices of three or more photons. The errors on the absolute BR's are dominated by the uncertainty on the value of $\tau_{L}$, which enters into the calculation of the geometrical efficiency. This source of uncertainty can be all but removed (at the cost of correlating the errors among the BR measurements) by applying the constraint that the $K_{L}$ BR's sum to unity. The sum of the four KLOE BR measurements plus the PDG BR values for $K_{L}$ decays to $\pi^{+} \pi^{-}, \pi^{0} \pi^{0}$, and $\gamma \gamma$ is $1.0104 \pm 0.0076$. Applying the constraint gives $\operatorname{BR}(\pi e v(\gamma))=0.4007 \pm 0.0006 \pm 0.0014, \operatorname{BR}(\pi \mu \nu(\gamma))=0.2698 \pm 0.0006 \pm 0.0014, \operatorname{BR}\left(3 \pi^{0}\right)=$ $0.1997 \pm 0.0005 \pm 0.0019$, and $\operatorname{BR}\left(\pi^{+} \pi^{-} \pi^{0}(\gamma)\right)=0.1263 \pm 0.0005 \pm 0.0011$, as well as a value for the $K_{L}$ lifetime, $\tau_{L}=50.72 \pm 0.17 \pm 0.33 \mathrm{~ns}$.

KLOE has also measured $\tau_{L}$ directly, using $10^{7} K_{L} \rightarrow 3 \pi^{0}$ events from $400 \mathrm{pb}^{-1}$ of 2001-2002 data [15]. $K_{L} \rightarrow \pi^{+} \pi^{-} \pi^{0}$ decays, for which the track and photon vertices can be independently reconstructed, provide a control sample for checking the EmC time scale, and demonstrate that the efficiency for reconstructing a vertex of three or more photons is uniformly greater than $99 \%$ inside the fiducial volume $\left(0.37 \lambda_{L}\right)$. The result, $\tau_{L}=50.92 \pm 0.17 \pm 0.25 \mathrm{~ns}$, is consistent with the value obtained from the sum of the $K_{L}$ BR's. These are two independent measurements; their average gives the KLOE value, $\tau_{L}=50.84 \pm 0.23$ ns. For comparison, the best previous measurement is $\tau_{L}=51.54 \pm 0.44 \mathrm{~ns}[16]$.

The form-factor slopes for semileptonic kaon decays are another important input for the determination of $V_{u s}$. Using a sample of $328 \mathrm{pb}^{-1}$ of 2001-2002 data, KLOE has obtained the distribution in $t \equiv\left(p_{K}-p_{\pi}\right)^{2} / m_{\pi^{+}}^{2}$ for $2 \times 10^{6} K_{L} \rightarrow \pi e v$ decays. The data are divided into subsamples by run period and lepton charge, and the $t$ distributions are fit with the forms $f_{+}(t)=$ $f_{+}(0)\left[1+\lambda_{+} t\right]$ and $f_{+}(0)\left[1+\lambda_{+}^{\prime} t+\lambda_{+}^{\prime \prime} t^{2} / 2\right]$. The linear fit gives $\lambda_{+}=(28.6 \pm 0.5 \pm 0.8) \times 10^{-3}$ with $\chi^{2} / N_{\text {dof }}=330 / 363$, while the quadratic fit gives $\lambda_{+}^{\prime}=(25.5 \pm 1.5 \pm 1.9) \times 10^{-3}$ and $\lambda_{+}^{\prime \prime}=$ $(1.4 \pm 0.7 \pm 0.7) \times 10^{-3}$, with correlation coefficient $\rho\left(\lambda_{+}^{\prime}, \lambda_{+}^{\prime \prime}\right)=-0.95$ and $\chi^{2} / N_{\text {dof }}=325 / 362$. 
These results are preliminary. The additional statistics from 2004-2005 running should help in gauging the significance of the quadratic term, as well as with the measurement of the form-factor slopes for $K_{L} \rightarrow \pi \mu \nu$ decays.

\section{Conclusions}

The KLOE 2001-2002 data have yielded measurements of the BR's for the $K_{e 3}$ decay of the $K_{S}$, the $K_{e 3}$ and $K_{\mu 3}$ decays of the $K_{L}$, and the $K_{L}$ lifetime. As described elsewhere in these proceedings [17], these results, together with preliminary measurements of the BR's for the semileptonic decays of charged kaons, lead to the determination $\left|V_{u s} f_{+}(0)\right|=0.2170 \pm 0.0005$, with an uncertainty of just $0.25 \%$. KLOE has also used the 2001-2002 data to obtain the first measurement of the semileptonic asymmetry $A_{S}$ and the most stringent limit on $\operatorname{BR}\left(K_{S} \rightarrow 3 \pi^{0}\right)$. In addition to statistical improvements on the above results, the data from 2004-2005 will allow new results on the BR's for $K_{S}$ decays to $\pi \mu \nu$ and $\pi^{+} \pi^{-} \pi^{0}$, the form-factor slopes for $K_{e 3}$ and $K_{\mu 3}$ decays of the $K_{L}$, and the first studies of the $K_{S} K_{L}$ system via interference in the relative decay-time spectrum.

\section{References}

[1] M. Adinolfi et al., Nucl. Instrum. Meth. A, 488 (2002) 51.

[2] M. Adinolfi et al., Nucl. Instrum. Meth. A, 482 (2002) 364.

[3] F. Ambrosino et al., Nucl. Instrum. Meth. A, 534 (2004) 403.

[4] C. Gatti, hep-ph/0507280.

[5] C.D. Buchanan et al., Phys. Rev. D, 45 (1992) 4088.

[6] Particle Data Group, S. Eidelman et al., Phys. Lett. B, 592 (2004) 1.

[7] R.A. Bertlmann, W. Grimus, and B.C. Hiesmayr, Phys. Rev. D, 60 (1999) 114032.

[8] T. Spadaro for the KLOE Collaboration, Response of the KLOE Electromagnetic Calorimeter to Low-Energy Particles, in proceedings of 11 th International Conference on Calorimetry in Particle Physics (Calor 2004), Perugia, Italy, 2004.

[9] V. Cirigliano et al., Eur. Phys. J. C, 33 (2004) 369.

[10] KLOE Collaboration, A. Aloisio et al., Phys. Lett. B, 538 (2002) 21.

[11] KLOE Collaboration, F. Ambrosino et al., Phys. Lett. B, 619 (2005) 61.

[12] KLOE Collaboration, A. Aloisio et al., Phys. Lett. B, 535 (2002) 37.

[13] KTeV Collaboration, T. Alexopoulos et al., Phys. Rev. D, 70 (2004) 092006.

[14] KLOE Collaboration, F. Ambrosino et al., Phys. Lett. B, in press [hep-ex/0508027].

[15] KLOE Collaboration, F. Ambrosino et al., Phys. Lett. B, 626 (2005) 15.

[16] K.G. Vosburgh et al., Phys. Rev. D, 6 (1972) 1834.

[17] B. Sciascia for the KLOE Collaboration, KLOE extraction of $V_{u s}$ from kaon decays and lifetimes, these proceedings [PoS(HEP2005)287]. 